Classification

Physics Abstracts

$61.10 \mathrm{~F}-73.60 \mathrm{~F}-61.70 \mathrm{R}-68.55 \mathrm{~B}$

\title{
Caractérisation par diffraction $X$ des superréseaux GaAlAs-GaAs. Importance des techniques et de la procédure utilisées
}

\author{
P. Auvray, M. Baudet, J. Caulet et A. Regreny \\ Centre National d'Etudes des Télécommunications, LAB/OCM/MPA, Route de Trégastel, 22301 Lannion \\ Cedex, France
}

(Reçu le 4 novembre 1988, révisé le 1er mars 1989, accepté le 24 mars 1989)

\begin{abstract}
Résumé. - Les techniques de diffraction $\mathrm{X}$ sont à l'heure actuelle largement répandues pour déterminer les paramètres structuraux des superréseaux (SR) à base de semi-conducteurs. Elles permettent en outre de détecter certaines particularités de ces structures regroupées sous le terme "défauts » tels que gradients latéraux de période et de composition, fluctuations d'épaisseur dans la direction de la croissance, dislocations, courbure et désorientation du substrat, à condition d'utiliser les appareils et les procédures adaptés. Ces défauts et leurs effets sur les diagrammes de rayons $X$ sont décrits ainsi que les moyens permettant de les mettre en évidence.

Abstract. - X-ray diffraction techniques are at the present time widely used to determine the structural parameters of artificial semiconductor based superlattices (SL). They can also reveal most of the peculiarities, or «defects", of these structures, namely lateral and/or vertical variations of composition and thicknesses, dislocations, substrate warpage and misorientation provided the right set-ups and procedures are used. These defects and their effects on the X-ray diagrams are described together with the most adequate experiments to bring them into view.
\end{abstract}

\section{Introduction.}

Les superréseaux de composition fabriqués par épitaxie par jets moléculaires (EJM) présentent des inhomogénéités structurales qui, par leur caractère systématique, doivent être considérées comme des paramètres qu'il convient donc de déterminer au même titre que les épaisseurs et les compositions des couches constituant la super-période. Cette détermination est en général effectuée au moyen de la diffraction des rayons $\mathrm{X}$; or, on constate que ces défauts se manifestent sensiblement de la même manière sur la forme et la position des réflexions caractéristiques de ces structures. Il en résulte que :

- ̀̀ un même effet peut correspondre plusieurs causes..

- à l'inverse, une seule cause peut expliquer plusieurs effets...

L'interprétation des diagrammes expérimentaux en vue de décrire le matériau réel avec ses inhomogénéités, nécessite donc d'avoir au préalable, réperto- rié tous les types d'écarts à la structure idéale et d'en avoir évalué séparément les conséquences au niveau de la diffraction. Il est d'autre part indispensable que tous les facteurs expérimentaux susceptibles de modifier ces profils et d'en compliquer l'interprétation soient également estimés et, si possible, éliminés...

Après un rappel de la structure idéale de référence d'un SR de composition, une revue sera faite des deux principaux types de défauts structuraux classiquement rencontrés dans les SR fabriqués par EJM en insistant plus particulièrement sur celui dont les conséquences sur les profils de diffraction sont les plus catastrophiques, à savoir l'angle de coupe, volontairement introduit ou non. Les principales caractéristiques des appareils visant à éliminer les causes expérimentales de perturbation des profils seront ensuite décrites. Enfin, le détail d'une procédure expérimentale destinée en particulier à s'affranchir de l'effet géométrique dû à l'angle de coupe, sera donné à titre de conclusion. 


\section{Les écarts à la structure idéale et leurs effets.}

Un SR idéal GaAlAs-GaAs est constitué de deux types de couches d'épaisseur et de composition constantes, épitaxiées alternativement et de façon cohérente sur un substrat sans défaut dont la surface est un plan cristallographique d'indices simples, (001) par exemple (Fig. 1) :

- chaque strate est constituée d'un nombre de couches moléculaires complètes;

- les interfaces entre les diverses strates sont réduites à un plan atomique délimitant de façon abrupte la transition d'un matériau à l'autre ;

- le SR est supposé identique à lui-même en tous points de la surface de l'échantillon.
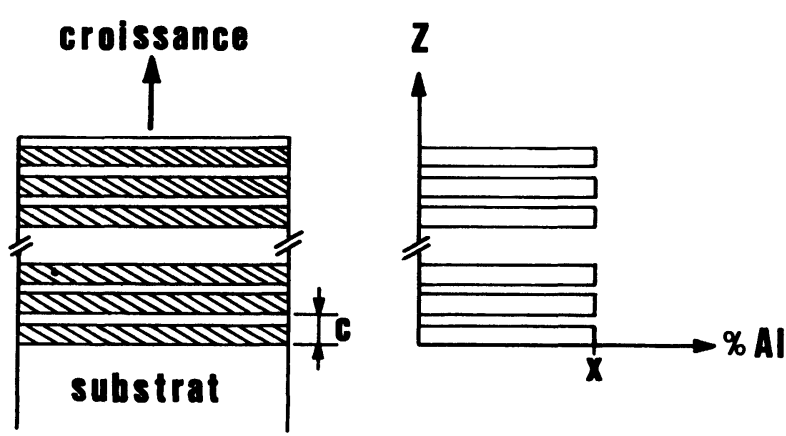

Fig. 1. - Structure idéale d'un superréseau de composition. $C=$ période du superréseau. $Z=$ direction de croissance. $x=$ composition en aluminium des couches de $\mathrm{Ga}_{(1-x)} \mathrm{Al}_{x} \mathrm{As}$.

[Ideal structure of a composition modulated superlattice. $C=$ superlattice period. $Z=$ growth direction. $x=$ aluminium composition of the $\mathrm{Ga}_{(1-x)} \mathrm{Al}_{x} \mathrm{As}$ layers.]

Les SR réels présentent par rapport à cette structure idéale, des écarts provenant soit du processus de croissance soit des caractéristiques du substrat. Par la suite, seuls ont été envisagés les défauts réellement identifiés par diffraction $X$ ou par d'autres méthodes d'analyse telles que la microscopie électronique par transmission (MET) classique ou en mode haute résolution, l'analyse ionique secondaire (SIMS), la spectroscopie de photoluminescence (PL) et l'excitation de la photoluminescence (PLE).

2.1 ECARTS LIÉS A LA CROISSANCE. - Dans le tableau I sont répertoriés les principaux défauts liés au processus de croissance, à savoir l'épitaxie par jets moléculaires, avec leur origine précise et les effets qu'ils produisent sur les profils de diffraction (sur les réflexions du réseau moyen d'une part, et sur les pics satellites, d'autre part).

2.1.1 Les gradients latéraux de composition et d'épaisseur [1]. - Ces défauts liés à la géométrie du bâti (nature du porte-substrat et configuration des cellules d'évaporation) provoquent des élargissements de pics dont l'importance dépend de la dimension et de la position de l'impact du faisceau d'analyse sur l'échantillon : sont concernés les pics du réseau moyen et tous les satellites d'ordre différent de zéro. On met en évidence ces gradients en utilisant un faisceau d'analyse de faible section (Fig. 2).

2.1.2 Les variations d'épaisseur ou de composition dans la direction de croissance [2]. - Elles ont pour origine une dérive d'une ou de plusieurs conditions de croissance et provoquent suivant leur amplitude, des modifications des profils de diffraction qui vont du simple élargissement à des structures plus complexes composées de contributions distinctes (Fig. 3). Seules sont affectées de façon notable les réflexions satellites, indépendamment de la dimension de l'impact et de la position du faisceau d'analyse sur l'échantillon.

2.1.3. La présence inopinée de couches non prévues dans la séquence de croissance [3]. - Ce type de défauts, moins fréquents que les précédents et cependant d'origine similaire, provient de la variation soudaine d'un paramètre de croissance qui reprend immédiatement après sa valeur nominale (ex.: défaillance dans le fonctionnement d'un cache). Il se manifeste, dans une structure régulière, par l'apparition de couches différentes et non prévues dans la séquence de croissance. Dans l'exemple choisi (Fig. 4), la présence d'un puits élargi dans une séquence parfaitement périodique provoque le dédoublement (splitting) de tous les pics satellites et modifie la structure des oscillations associées aux réflexions intenses, oscillations dont la période est sans relation apparente avec un élément connu de la séquence. Ces effets ne dépendent ni de la position ni de la taille du faisceau d'analyse.

2.1.4 Les fluctuations d'épaisseur des couches ou désordre résiduel [3]. - Ce défaut est inhérent au processus de croissance puisqu'il est surtout lié à la maîtrise des mouvements des caches dont dépendent la précision et la reproductibilité des temps de dépôt des couches successives, c'est-à-dire de leurs épaisseurs. Pas d'influence sur les pics du réseau moyen, par contre, suivant l'importance de cette fluctuation aléatoire et la valeur de la période du SR, on observe des effets qui vont du simple élargissement à la base des pics satellites à l'apparition de pics subsidiaires distribués autour des positions théoriques des pics satellites de la structure correspondante supposée parfaitement régulière (Fig. 5) : ces effets qui ne concernent que la stricte périodicité du SR, sont pratiquement insensibles à la taille et à la position de l'impact du faisceau d'analyse.

2.1.5 La rugosité des interfaces [4]. - Elle résulte à la fois des conditions thermodynamiques de crois- 
Tableau I. - Défauts structuraux liés à la croissance.

[Structural defects related to the growth process.]

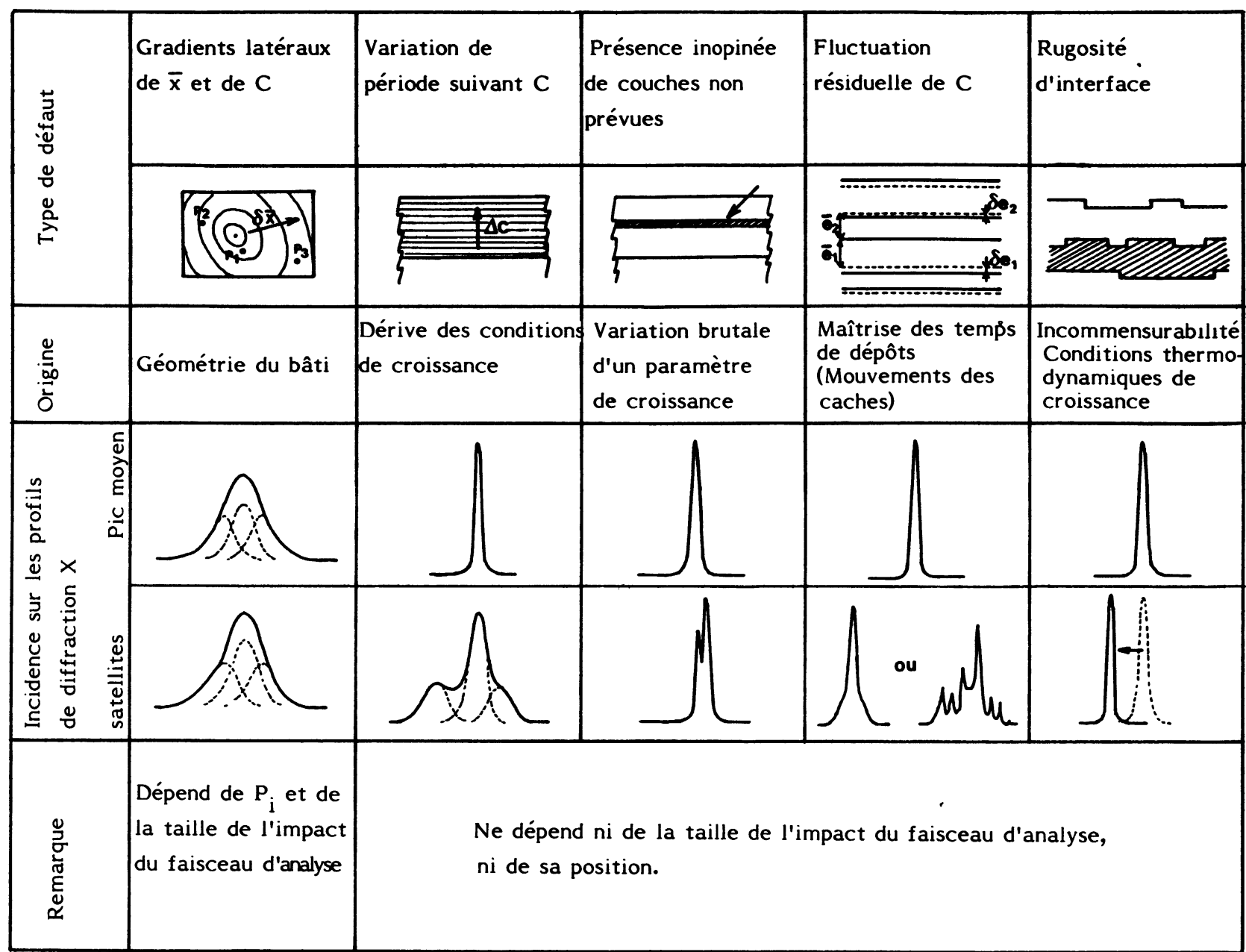

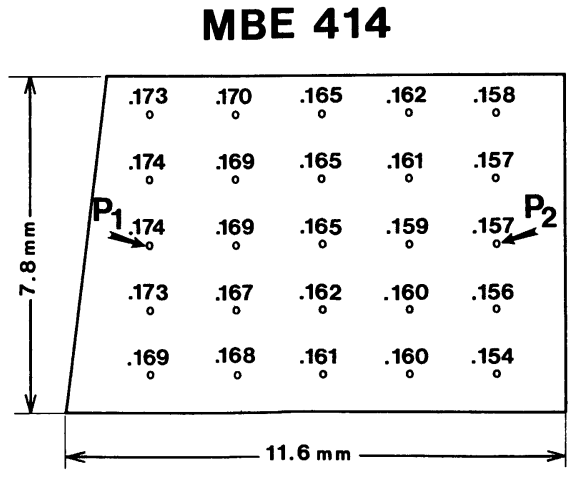

a)

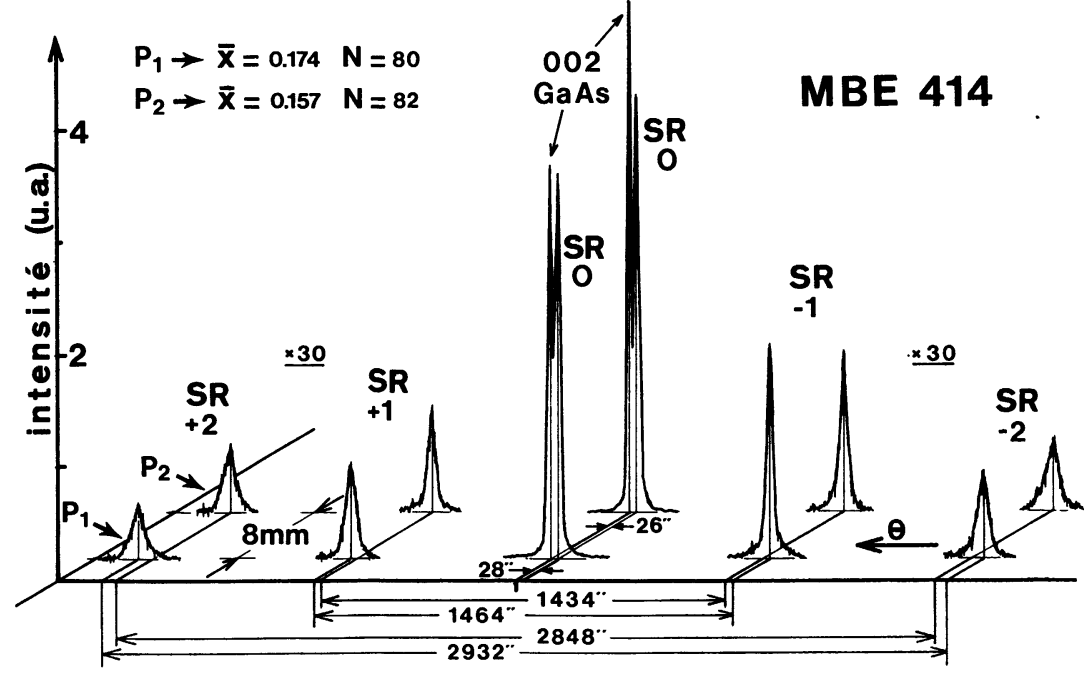

b)

Fig. 2. - Echantillon MBE 414 : a) cartographie de $\bar{x}$ et b) diagrammes de diffraction obtenus au voisinage de la réflexion GaAs 002 aux points $P_{1}$ et $P_{2}$, distants de $8 \mathrm{~mm}$. Section du faisceau d'analyse : $D_{\mathrm{f}}=0,1 \times 0,6 \mathrm{~mm}^{2}$.

[Sample MBE 414: a) $\bar{x}$ mapping and b) diffraction diagrams obtained close to the GaAs 002 reflection at points $\mathrm{P}_{1}$ and $\mathrm{P}_{2}, 8 \mathrm{~mm}$ apart. Incident beam section : $D_{\mathrm{f}}=0.1 \times 0.6 \mathrm{~mm}^{2}$.] 


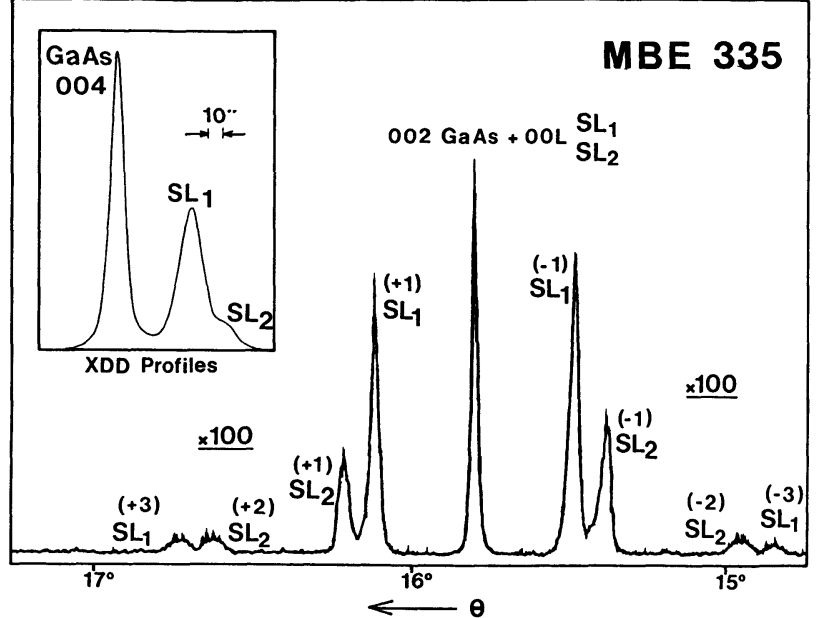

a)

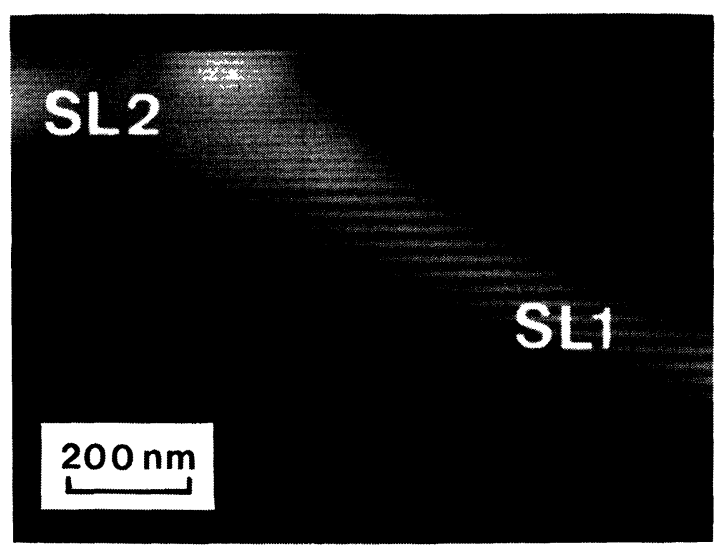

b)

Fig. 3. - Echantillon $M B E 335$ : a) profils de diffraction $\mathrm{X}$ et b) image de microscopie électronique par transmission en champ sombre d'une section (110) montrant la présence de deux superréseaux (SL1 et SL2) de période et de composition différentes. Le passage d'un mode de croissance stabilisé As (SL1) à un mode stabilisé Ga (SL2) fait passer la période et la composition respectivement de $C_{1}=226 \AA$ et $x_{1}=0,298$ à $C_{2}=128 \AA$ et $x_{2}=0,370$.

[Sample MBE 335 : a) X-ray diffraction profiles and b) transmission electron microscopy (110) cross-section dark-field image showing the presence of two superlattices (SL1 and SL2) with different period and composition. The change from an As stabilized growth mode (SL1) to a Ga stabilized one (SL2) leads to changes in period and composition from $C_{1}=226 \AA$ and $x_{1}=0.298$ to $C_{2}=128 \AA$ and $x_{2}=0.370$ respectively].

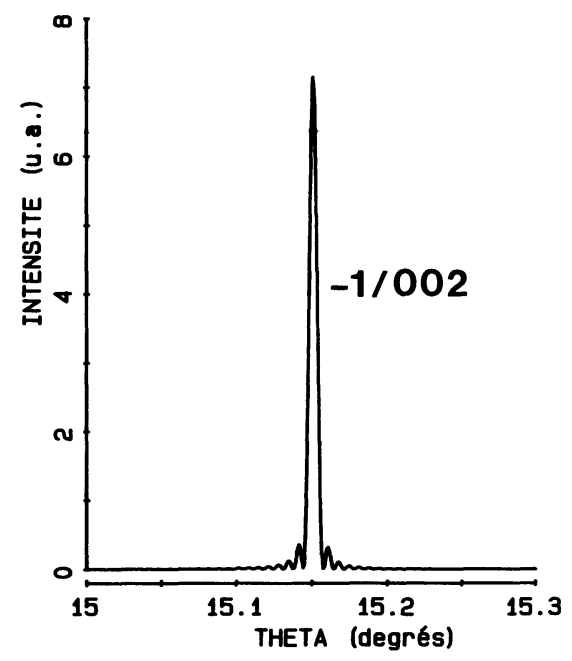

a)

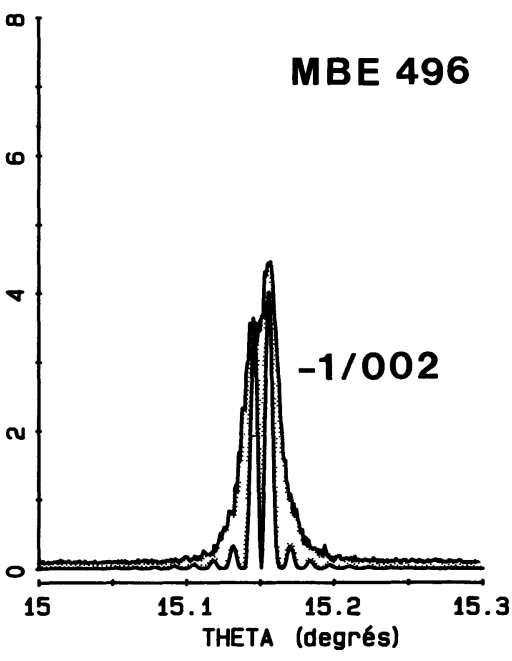

b)

Fig. 4. - Echantillon MBE 496 ( $S=0$ dans Ref. [3]). Superréseau : $\operatorname{GaAlAs}(34,8 \AA) / \operatorname{GaAs}(34,8 \AA), x=0,262$. Satellite - 1/002 a) profil calculé correspondant à la structure régulière (99 périodes), b) profils observé et calculé de la même structure comportant un puits élargi, $W_{\mathrm{e}}=67,6 \AA$ (49 périodes +1 période avec $W_{\mathrm{e}}+49$ périodes).

[Sample MBE 496 ( $S=0$ in Ref. [3]). Superlattice: $\operatorname{GaAlAs}(34.8 \AA) / G a A s(34.8 \AA$ ),$x=0.262$. Satellite $-1 / 002$ a) calculated profile corresponding to the regular structure ( 99 periods), b) observed and calculated profiles corresponding to the same structure with an enlarged well, $W_{\mathrm{e}}=67.6 \AA$ (49 periods +1 period with $W_{\mathrm{e}}+49$ periods)].

sance et de la valeur non entière du nombre $\left(n_{i}\right)$ de plans moléculaires contenus dans chaque strate $(i)$ déposée. Cette incommensurabilité a pour principal effet de déplacer les pics satellites sans modification sensible de leur forme [3] et ce, en relation avec la valeur exacte de la période (Fig. 6). Comme dans les trois cas précédents, l'influence de ce défaut sur les profils de diffraction est indépendante de la forme et de la taille de la zone d'échantillon analysée. 


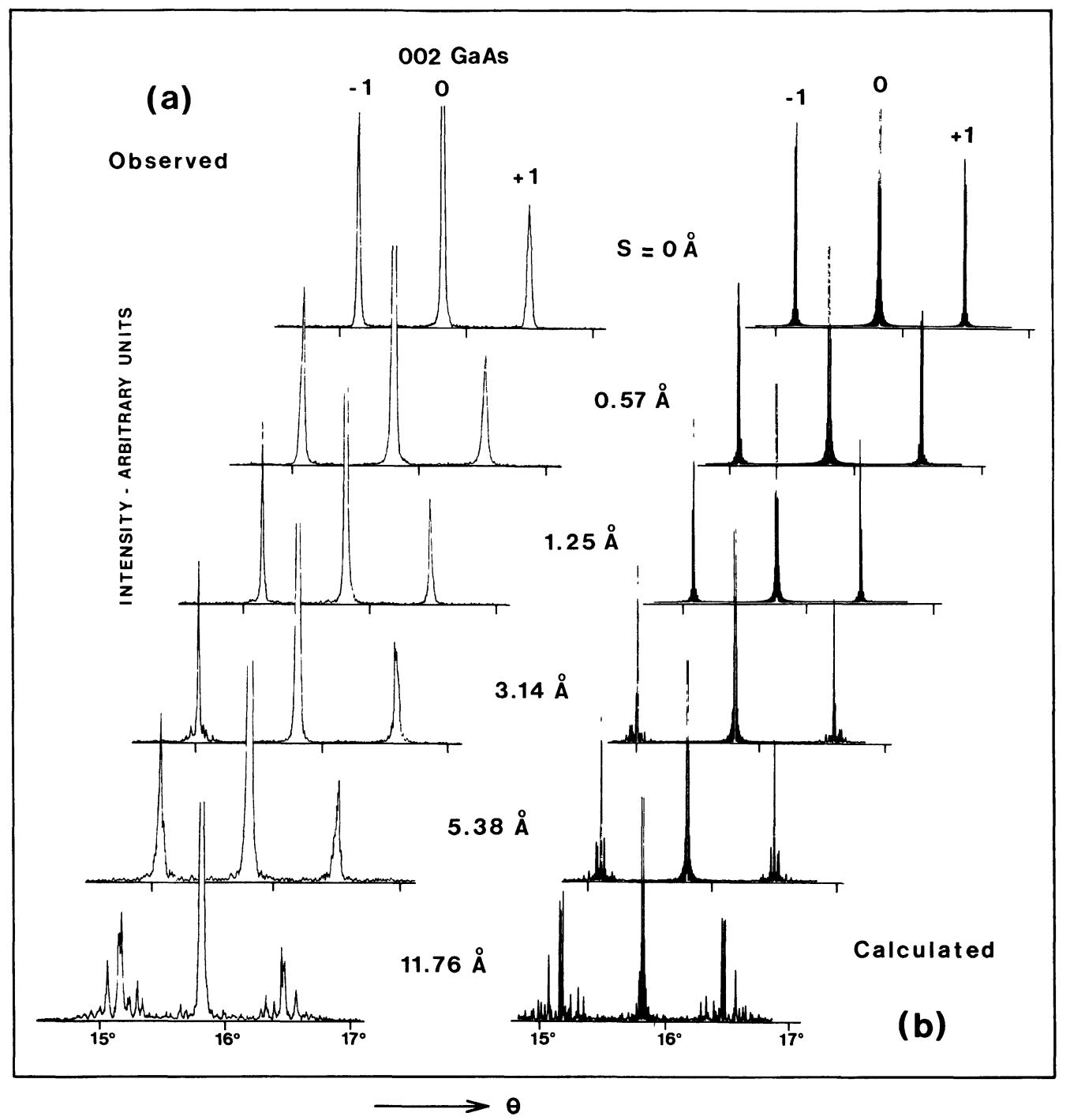

Fig. 5. - (Fig. 2 dans Ref. [3]). Diagrammes de diffraction a) observés et b) calculés, obtenus en couplage $\theta / 2 \theta$ au voisinage de la réflexion 002 du substrat montrant l'évolution des profils des pics satellites d'ordre \pm 1 en fonction d'un désordre introduit intentionnellement dans les épaisseurs des puits de GaAs, suivant une distribution gaussienne d'écart type $S$.

[(Fig. 2 in Ref. [3]). a) observed and b) calculated $\theta / 2 \theta$ diffraction diagrams close to the GaAs 002 substrate reflection showing the changes in the profiles of \pm 1 order satellite peaks as a function of an intentional GaAs well width disorder characterized by a gaussian distribution with standard deviation $S$.]

Fig. 6. - (Fig. 9 dans Ref. [3]). Profils de diffraction correspondant au satellite $-1 / 002$ calculés pour a) un superréseau GaAlAs-GaAs de période incommensurable $\left(n_{1}=n_{2}=12,31\right)$, b) un superréseau GaAlAs-GaAs de période commensurable $\left(n_{1}=n_{2}=12\right)$.

[(Fig. 9 in Ref. [3]). - 1/002 diffraction profiles calculated for a) a GaAlAs-GaAs superlattice with an incommensurate period $\left(n_{1}=n_{2}=12.31\right)$, b) a GaAlAs-GaAs superlattice with a commensurate period $\left.\left(n_{1}=n_{2}=12\right)\right]$.

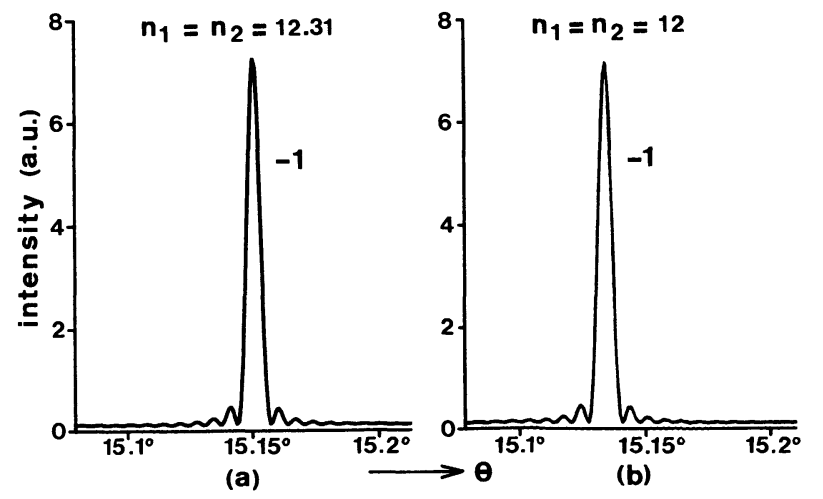


2.2 ECARTS liÉs AU SUbSTRAT. - Il s'agit de défauts liés à des caractéristiques intrinsèques au matériau ou à sa préparation, à l'exclusion de ceux dus à un nettoyage insuffisant de la surface ou à un mauvais collage du substrat dont les conséquences sur la croissance sont telles que toute caractérisation structurale devient inutile... Les principales causes d'altération des profils de diffraction relevant de cette catégorie sont au nombre de trois (Tab. II).

2.2.1 La qualité cristalline. - Généralement bonne pour les substrats de type laser, elle ne produit d'effets notables sur la largeur à mi-hauteur des pics que pour des densités de dislocations $\geqslant 10^{3} \mathrm{~cm}^{-2}$ (cas de certains substrats SI). La présence de dislocations dans le substrat dont certaines se propagent à l'interface et/ou dans la couche épitaxiée, provoque un élargissement des pics de diffraction du substrat et du réseau moyen, avec un éventuel rapprochement des deux pics en cas de relaxation totale ou partielle des contraintes dans le système (Fig. 7) ; parallèlement, on observe un élargissement de tous les pics satellites. Si la densité de dislocations est homogène dans tout l'échantillon, les mêmes effets sont observés quelles que soient la taille et la position de l'impact du faisceau d'analyse.

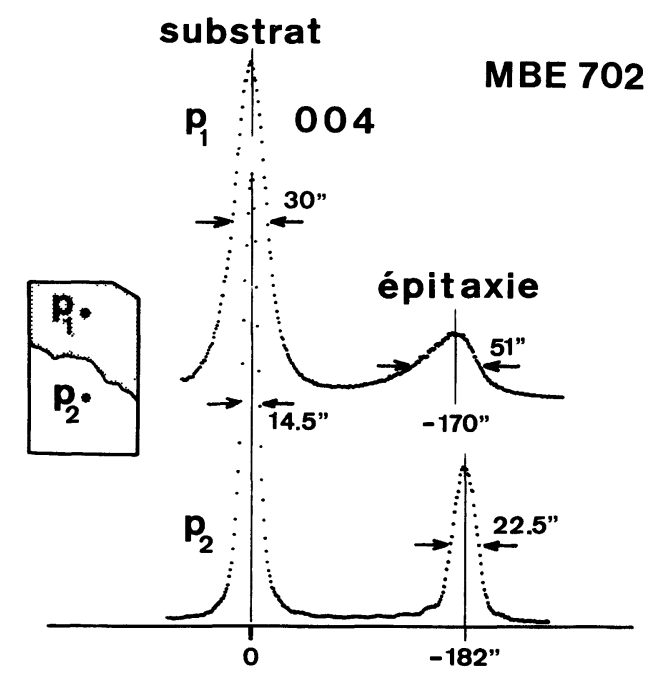

Fig. 7. - Echantillon MBE 702. Profils de double diffraction au voisinage de la réflexion 004 du substrat réalisés en deux points de l'échantillon. En $\mathrm{P}_{1}$ la densité de dislocations est supérieure à $10^{5} \mathrm{~cm}^{-2}$, en $\mathrm{P}_{2}$ elle est de l'ordre de $2 \times 10^{2} \mathrm{~cm}^{-2}$.

[Sample MBE 702. Double crystal diffraction profiles close to the $004 \mathrm{GaAs}$ substrate reflection obtained at points $P_{1}$ and $P_{2}$. At point $P_{1}$ the dislocations density is greater than $10^{5} \mathrm{~cm}^{-2}$, at point $P_{2}$ it is of the order of $2 \times 10^{2} \mathrm{~cm}^{-2}$.]

Tableau II. - Défauts structuraux liés au substrat.

[Structural defects related to the substrate.]

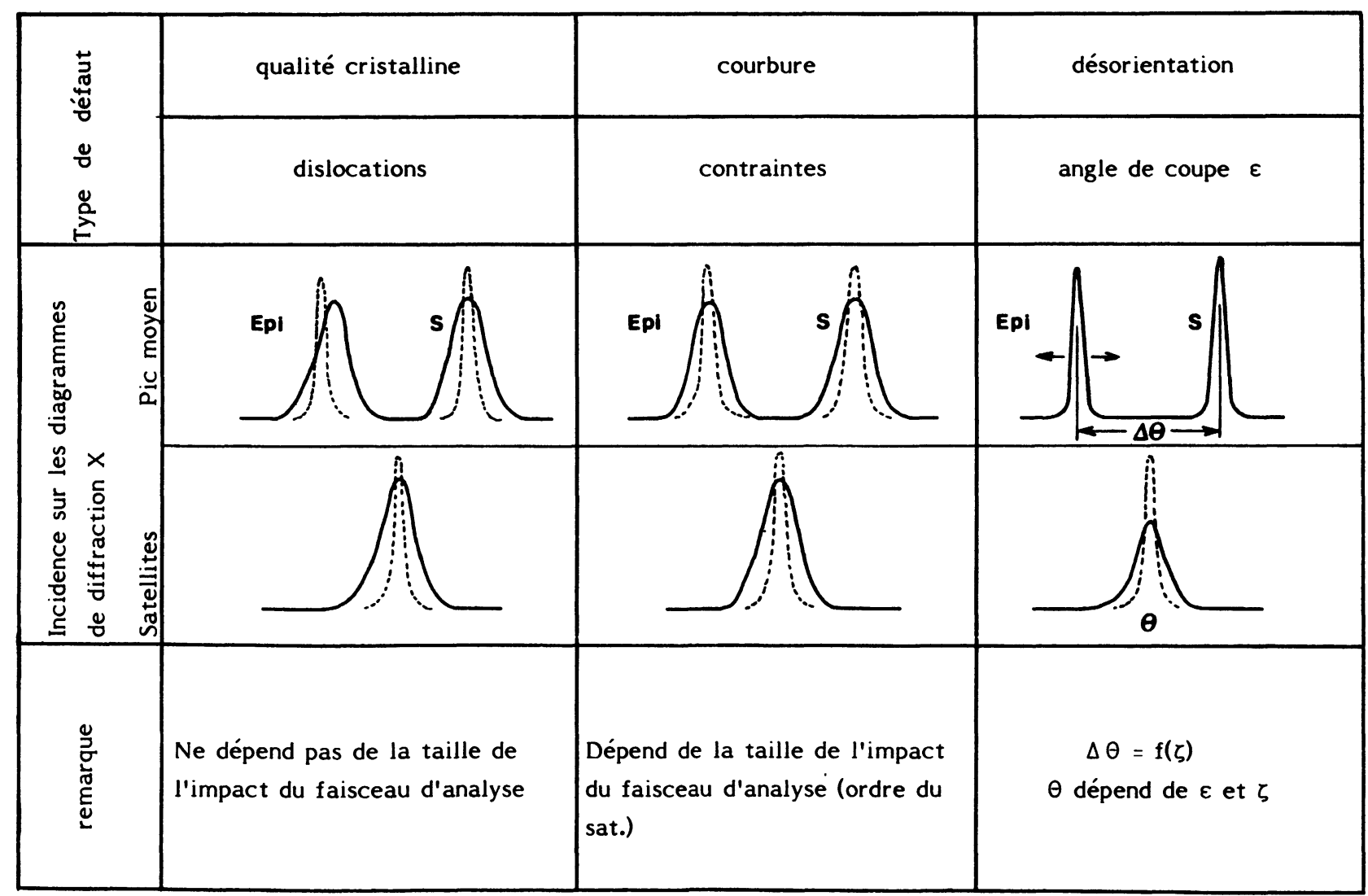


2.2.2 La courbure [5]. - C'est la conséquence de plusieurs effets conjugués, à savoir la courbure initiale du substrat due à un état de surface différent de ses deux faces, l'état contraint du système substrat/épitaxie et la présence d'indium sur la face arrière [6]. Elle a pour effet d'élargir tous les pics et ce, d'autant plus que la courbure est grande et la surface analysée étendue (Fig. 8).

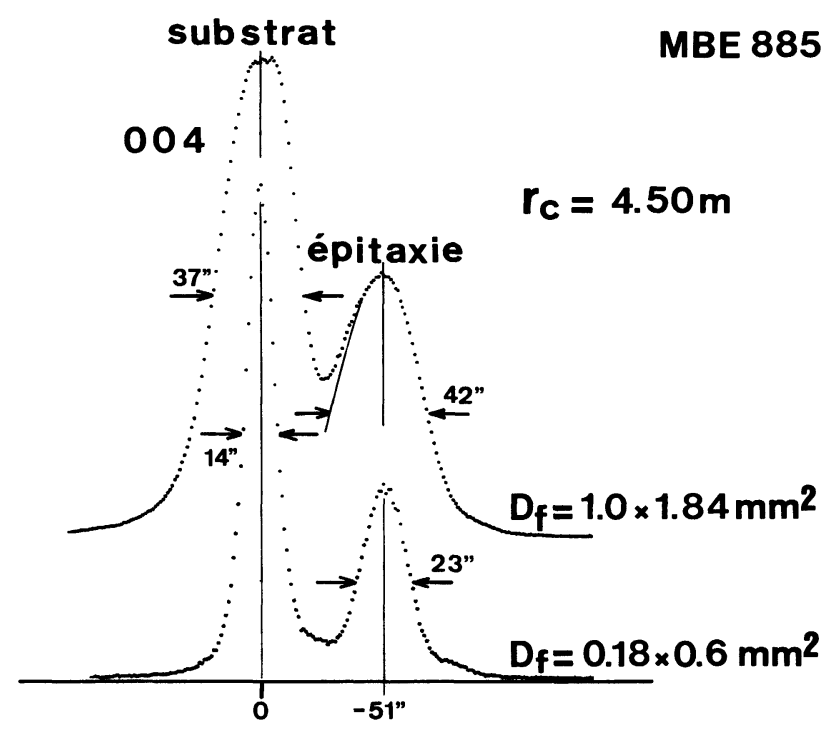

Fig. 8. - Echantillon $M B E$ 885. Rayon de courbure $r_{\mathrm{c}}=4,50 \mathrm{~m}$. Profils de double diffraction au voisinage de la réflexion 004 du substrat obtenus au même endroit pour deux tailles de l'impact du faisceau d'analyse : $D_{\mathrm{f}}=1,0 \times$ $1,84 \mathrm{~mm}^{2}$ et $0,18 \times 0,6 \mathrm{~mm}^{2}$.

[Sample MBE 885. Radius of curvature $r_{\mathrm{c}}=4.50 \mathrm{~m}$. Double crystal diffraction profiles close to the $004 \mathrm{GaAs}$ substrate reflection obtained from the same area for two incident beam sizes : $D_{\mathrm{f}}=1.0 \times 1.84 \mathrm{~mm}^{2}$ and $0.18 \times$ $0.6 \mathrm{~mm}^{2}$.]

2.2.3 La désorientation [7, 8]. - Qu'un angle soit volontairement introduit ou non lors de l'orientation préliminaire d'une plaquette avant sa découpe, ses faces ne sont jamais strictement parallèles à un plan atomique dense, (001) par exemple. En fait, il existe toujours une désorientation $(\varepsilon)$ qui dépend de la précision avec laquelle cette opération est exécutée. Aussi faible soit-elle $\left(\approx 15^{\prime}\right)$, la désorientation a des effets sensibles sur les positions et sur les intensités des pics satellites, indépendamment des caractéristiques géométriques du faisceau d'analyse.

Pour expliquer ces effets, il faut considérer le mécanisme de croissance des SR sur une face vicinale et montrer en quoi la présence d'un angle de coupe $(\varepsilon)$ modifie la forme du réseau cristallin de l'épitaxie.

Le plan d'épitaxie est a priori une surface présentant des gradins (marches atomiques) dont l'espacement est fonction de l'angle de coupe. Dans un but de simplification on supposera par la suite que toutes les marches ont la même longueur $\ell=d / \operatorname{tg} \varepsilon$ (Fig. 9). Dans des conditions de croissance telles

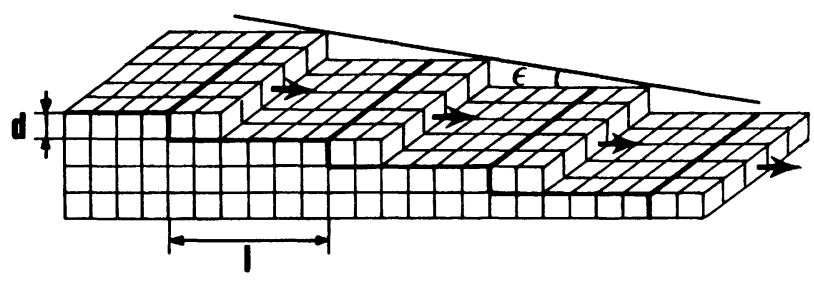

Fig. 9. - Représentation schématique d'une face vicinale. Les flèches matérialisent le déplacement du front de croissance, $\ell=$ longueur moyenne d'une marche de hauteur $d, \varepsilon=$ angle de désorientation $: \operatorname{tg} \varepsilon=d / \ell$.

[Schematic representation of a vicinal face. The arrows indicate the displacement of the growth front, $\ell=$ average length of a $d$-high step, $\varepsilon=$ misorientation angle : $\operatorname{tg} \varepsilon=d / \ell$.]

que, les longueurs de diffusion de surface des atomes de gallium et d'aluminium sont au moins égales à la longueur moyenne $\ell$ des marches [9], on peut considérer que le front de croissance est à tout moment identique à lui-même... En particulier, toutes les interfaces ont la même morphologie en gradins calquée sur celle de la surface du substrat de départ.

Si l'on se place dans l'hypothèse d'une épitaxie cohérente (Fig. 10), la distorsion qui assurera la continuité des réseaux de l'épitaxie et du substrat dans les trois dimensions résultera :

a) d'une déformation tétragonale dans la direction [001] des édifices cristallins situés au-dessus de chaque marche de façon à accorder leurs paramètres dans le plan des marches;

b) et d'un basculement $(\alpha)$ des plans atomiques (001) prolongeant ceux du substrat dans l'épitaxie, afin d'assurer la continuité des réseaux cristallins dans la direction perpendiculaire au plan des marches.

Il résulte de ce double mécanisme, une déformation monoclinique de la maille de l'épitaxie caractérisée par l'angle $\alpha$ dont l'expression est donnée par : $\operatorname{tg} \alpha=\operatorname{tg} \varepsilon .(\delta d / d)_{\perp} \quad$ ou $\quad(\delta d / d)_{\perp} \quad$ représente l'écart paramétrique mesuré perpendiculairement au plan des marches entre l'épitaxie et le substrat [10].

La figure 11 montre l'exemple d'un SR de courte période $(\mathrm{AlAs})_{3}(\mathrm{GaAs})_{3}$ épitaxié sur une face vicinale. Les couches d'AlAs sont déformées conformément au mécanisme précédemment décrit, par contre celles de GaAs ne subissent aucune déformation en dehors de la couche d'interface et ce, grâce à un mécanisme strictement inverse du précédent. Ainsi, si l'on considère dans cette structure les plans d'indices (00l) parallèles aux marches, dans les couches de GaAs ils sont parallèles à ceux du 


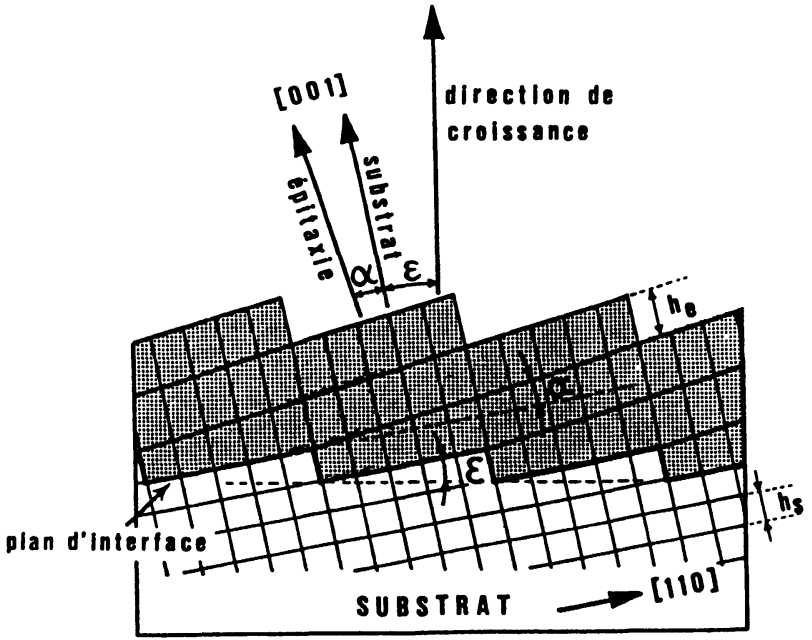

a)

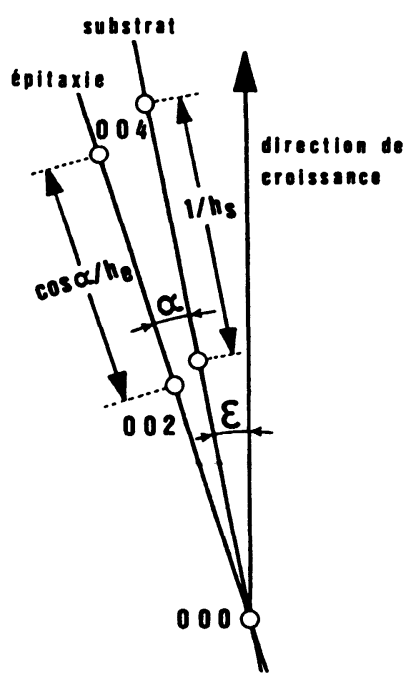

b)

Fig. 10. - Mécanisme de déformation du réseau cristallin d'une couche épitaxiée sur une face vicinale. Représentation dans l'espace réel (a) et dans l'espace réciproque (b), $\operatorname{tg} \alpha=\operatorname{tg} \varepsilon(\delta d / d)_{\perp}$ avec $(\delta d / d)_{\perp}=\left(h_{\mathrm{e}}-h_{\mathrm{s}}\right) / h_{\mathrm{s}}$.

[Deformation mechanism of the crystal lattice of a layer epitaxied on a vicinal plane. Real (a) and reciprocal (b) space representation, $\operatorname{tg} \alpha \operatorname{tg} \varepsilon=(\delta d / d) \perp \quad$ with $\left.(\delta d / d)_{\perp}=\left(h_{\mathrm{e}}-h_{\mathrm{s}}\right) / h_{\mathrm{s}}\right]$

substrat alors que dans les barrières, ils sont inclinés de l'angle $\alpha$.

La présence d'une périodicité supplémentaire dans le plan d'épitaxie nécessite la définition d'une nouvelle maille unitaire SR. Deux d'entre elles (équivalentes), $\mathrm{ABCD}$ et $\mathrm{ABC}^{\prime} \mathrm{D}^{\prime}$ sont représentées figure 11a dans l'espace réel projeté suivant la direction [1110]. Le réseau réciproque correspondant à ce SR (Fig. 11b) présente les caractéristiques suivantes.

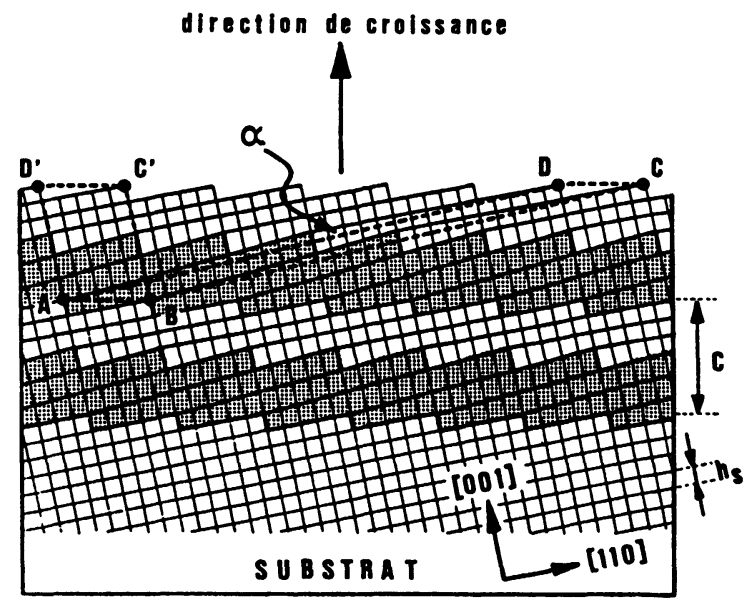

a)

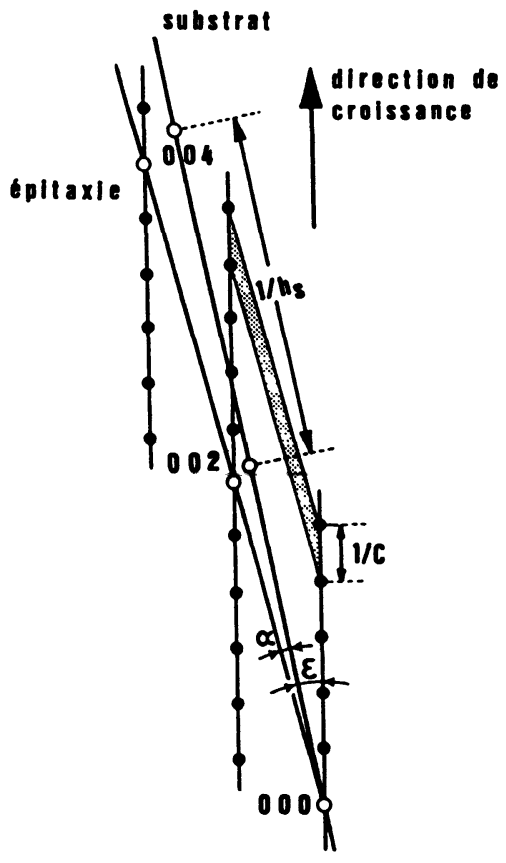

b)

Fig. 11. - Superréseau (AlAs $)_{3}(\mathrm{GaAs})_{3}$ épitaxié sur une face vicinale représenté dans l'espace réel (a) et dans l'espace réciproque (b). La direction de croissance normale au plan de l'échantillon, est confondue avec la direction de modulation de composition. $\mathrm{ABCD}$ et $\mathrm{ABC}^{\prime} \mathrm{D}^{\prime}$ sont 2 mailles unitaires équivalentes dans l'espace réel. $\operatorname{tg} \alpha=$ $\operatorname{tg} \varepsilon(\delta d / d)_{\perp}$ où $(\delta d / d)_{\perp}=\left(h_{\mathrm{e}}-h_{\mathrm{s}}\right) / h_{\mathrm{s}}$ avec $h_{\mathrm{s}}=d_{2}$ et $\bar{h}_{\mathrm{e}}=\left(n_{1} d_{1}+n_{2} d_{2}\right) /\left(n_{1}+n_{2}\right):$ l'indice 1 se réfère à AlAs et l'indice 2 à GaAs.

$\left[(\mathrm{AlAs})_{3}(\mathrm{GaAs})_{3}\right.$ superlattice epitaxied on a vicinal face, shown in real (a) and (b) reciprocal space. The growth axis, perpendicular to the sample surface, is the same as the composition modulation direction. $\mathrm{ABCD}$ and $\mathrm{ABC}^{\prime} \mathrm{D}^{\prime}$ are two equivalent unit cells in real space. $\operatorname{tg} \alpha=\operatorname{tg} \varepsilon(\delta d / d)_{\perp}$ where $(\delta d / d)_{\perp}=\left(h_{\mathrm{e}}-h_{\mathrm{s}}\right) / h_{\mathrm{s}}$ with $h_{\mathrm{s}}=d_{2}$ and $\hbar_{\mathrm{e}}=\left(n_{1} d_{1}+n_{2} d_{2}\right) /\left(n_{1}+n_{2}\right):$ index 1 refers to AlAs and index 2 to $\mathrm{GaAs}$.] 
- Les nœuds relatifs aux réflexions satellites du SR sont alignés sur des droites perpendiculaires à la surface de l'échantillon, alors que dans le modèle de déformation quadratique ils sont alignés dans la direction [001] du substrat.

- Les rangées $00 l$ du réseau moyen et du substrat ne sont pas confondues: elles forment un angle $\alpha$ défini comme précédemment, à ceci près que dans l'expression de $\operatorname{tg} \alpha$ appliquée aux SR, $(\delta \bar{d} / d)_{\perp}$ exprime l'écart paramétrique mesuré perpendiculairement entre le matériau moyen et le substrat.

- Les mailles primitives joignant les nœuds équivalents sont monocliniques: l'une d'entre elles, correspondant à la maille $\mathrm{ABCD}$ dans l'espace réel, est représentée sur la figure $11 \mathrm{~b}$.

L'enregistrement de cette section du réseau réciproque par la technique classique de balayage en couplage $\theta / 2 \theta$ consiste à décrire des droites qui toutes passent par l'origine. Par conséquent, il apparaît que seuls les satellites associés à l'origine pourront être enregistrés dans les conditions normales d'un balayage continu alors que tous les autres devront l'être individuellement, après un réglage préliminaire chaque fois différent. De même, les conditions d'exploration des réflexions $00 l$ du substrat seront différentes de celles du réseau moyen parce que portées par une autre rangée.

A titre d'exemple, la figure 12 présente deux diagrammes obtenus au voisinage de la réflexion 000 du même SR de courte période (AlAs) $)_{7}(\mathrm{GaAs})_{9}$ épitaxié sur un substrat (001) de type laser non intentionnellement désorienté : $\varepsilon=17^{\prime}$. Les conditions expérimentales sont strictement identiques, exception faite du réglage initial du goniomètre. Dans le premier cas (Fig. 12a), il a été réalisé en recherchant l'intensité maximale de la réflexion 002 GaAs (exploration de la rangée $00 l$ du substrat) alors que dans le deuxième cas (Fig. 12b), l'optimisation du réglage s'est effectuée en utilisant la réflexion satellite $+1 / 000$ (exploration de la rangée $00 \mathrm{~L} \mathrm{du}$ SR).

Entre ces deux diagrammes, il y a une différence de deux ordres de grandeur entre les échelles d'intensité : cet exemple met en évidence le fait qu'une désorientation, même très faible $\left(17^{\prime}\right)$, peut avoir une influence considérable sur la visibilité et l'intensité des réflexions satellites, si.elle n'est pas correctement prise en compte au niveau expérimental.

\section{Caractéristiques des montages expérimentaux.}

La complexité des diagrammes est telle que toute cause extérieure susceptible de les modifier doit être impérativement réduite ou mieux, supprimée. Quel que soit le montage expérimental utilisé $(\theta / 2 \theta$ ou DDX), deux points essentiels doivent faire l'objet d'une attention toute particulière : il s'agit du faisceau d'analyse et du porte-échantillon.

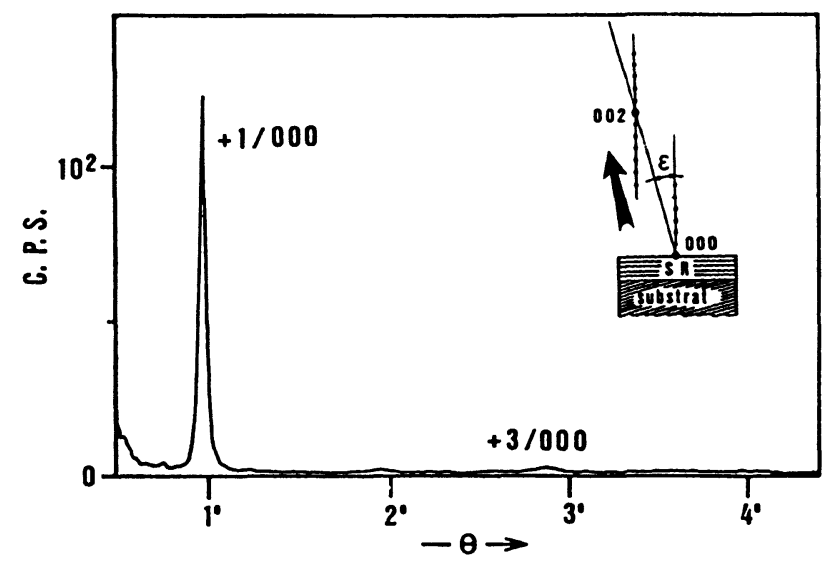

a)

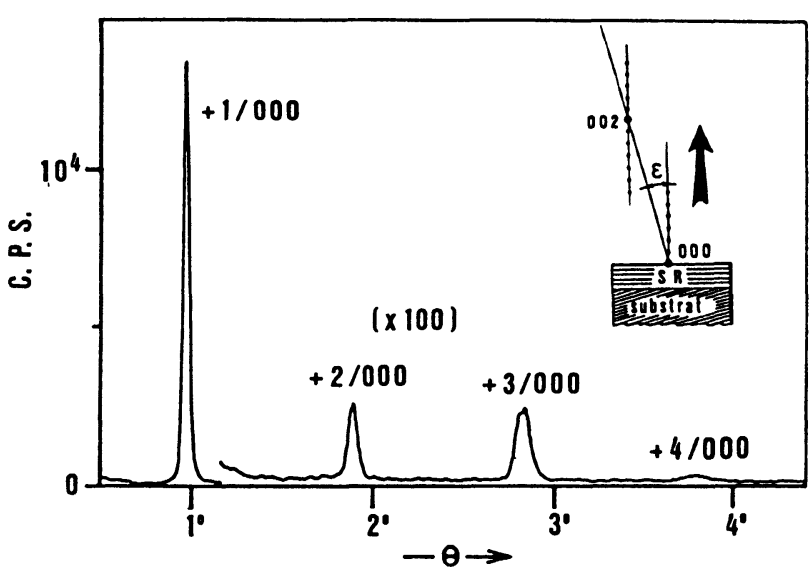

b)

Fig. 12. - Echantillon MBE 585. Superréseau (AlAs) $)_{7}(\mathrm{GaAs})_{9}$ épitaxié sur un substrat (001) non-intentionnellement désorienté $\left(\varepsilon=17^{\prime}\right)$. Enregistrements en couplage $\theta / 2 \theta$ au voisinage de l'origine. Optimisation $\mathrm{du}$ réglage initial du goniomètre, (a) sur la réflexion 002 GaAs (échelle : $10^{2} \mathrm{cps}$ ) et (b) sur le satellite $\mathrm{SR}+1 / 000$ (échelle : $10^{4} \mathrm{cps}$ ).

[Sample MBE 585. (AlAs) $)_{7}(\mathrm{GaAs})_{9}$ superlattice grown by epitaxy on a non-intentionally misoriented (001) GaAs substrate. $\theta / 2 \theta$ scans in the vicinity of the origin. Initial $\theta / 2 \theta$ coupling of the goniometer optimized (a) on the $002 \mathrm{GaAs}$ reflection (scale : $10^{2} \mathrm{cps}$ ) and (b) on the $+1 / 000$ satellite reflection (scale : $10^{4} \mathrm{cps}$ ).]

a) Il est indispensable de travailler en faisceau rigoureusement monochromatique et parallèle. De plus, sa section droite $(s)$ doit être aussi faible que possible afin de réduire au maximum la surface - d'échantillon analysée et permettre ainsi l'étude des gradients latéraux de période et de composition : $s \leqslant 0.2 \mathrm{~mm}^{2}$ pour un tube conventionnel à foyer fin fonctionnant à $1200 \mathrm{~W}$.

b) Le porte-échantillon doit être conçu de telle manière qu'il maintienne les substrats sans contrainte tout en rendant possible leur déplacement en translation et en rotation dans le plan perpendiculaire au 
plan d'incidence passant par l'axe de rotation du goniomètre.

Bien entendu, la comparaison des résultats nécessite que toutes les expériences soient réalisées à la même température.

\section{Procédures expérimentales.}

L'élimination de l'indium sur la face arrière de l'échantillon doit être considérée comme un préalable indispensable à toute caractérisation précise. Celle-ci doit être menée de la manière suivante :

1) détermination de l'angle de coupe du substrat et repérage de la direction d'intersection $(\Sigma)$ des plans réflecteurs avec la surface;

2) enregistrement en continu des satellites de la réflexion $000 \mathrm{du}$ SR dans le but de déterminer une valeur locale approchée de $n_{1}+n_{2}$ et de mettre en évidence les extinctions systématiques ;

3) $\Sigma$ étant placé perpendiculairement au plan d'incidence, enregistrement de chacun des satellites des réflexions 002 ou 004 du SR afin d'affiner la mesure précédente, la déformation monoclinique du réseau réciproque nécessitant pour chaque satellite un couplage $\theta / 2 \theta$ différent. Détermination de la composition moyenne locale $(\bar{x})$ au moyen des écarts angulaires des réflexions 002 et/ou 004 du substrat et de l'épitaxie, mesurés pour les deux positions de $\Sigma$ à $180^{\circ}$ l'une de l'autre ;

4) translation de l'échantillon dans son plan et retour à l'étape précédente pour établir des cartographies de $\bar{x}$ et de $n_{1}+n_{2}$ destinées à mettre en évidence des gradients latéraux de composition et de période.
Les enregistrements ainsi obtenus doivent permettre de résoudre la structure du SR avec ses inhomogénéités : il est cependant indispensable de confirmer la solution finalement retenue par des calculs de profils faisant intervenir tous les éléments déterminés par cette procédure.

\section{Conclusion.}

Les SR fabriqués par EJM présentent tous à des degrés divers des écarts à l'idéalité principalement dus :

- à la croissance (bâti, conditions thermodynamiques...);

- au substrat (orientation, nature, préparation...).

Leurs effets sur les diagrammes de diffraction $\mathrm{X}$ sont pratiquement les mêmes, à savoir :

- déplacements des pics ;

- modifications de leur profil.

En général, plusieurs défauts coexistent et leurs effets s'ajoutent. Il en résulte une difficulté dans l'appréciation de leur contribution respective et par conséquent des problèmes pour les identifier sans ambiguité.

La mise en évidence expérimentale de ces défauts implique :

— l'utilisation d'équipements adaptés ;

- le suivi d'une procédure prenant notamment en compte l'existence de l'angle $\varepsilon$. L'effet de cet angle de coupe est de loin le plus fort puisqu'il peut conduire à la disparition complète de satellites pourtant intenses : il faut donc veiller à s'en affranchir expérimentalement...

\section{Bibliographie}

[1] Talalaeff G., Thin Solid Films 150 (1987) 369-391.

[2] Poudoulec A., Guenais B., Auvray P., Baudet M. and Regreny A., Inst. Phys. Conf. Ser. Oxford 87 (1987) 213.

[3] Auvray P., Baudet M. and Regreny A., J. Appl. Phys. 62 (1987) 456.

[4] Deveaud B., Emery J. Y., Chomette A., LaMBERT B. and BAudet M., Appl. Phys. Lett. 45 (1984) 1078.

[5] Clec'h G. et Emery J. Y., Note Technique Interne CNET, NT/LAB/ICM/130 (1985).
[6] Petroff J. F., Sauvage-Simkin M., Bensoussan S., Capelle B., Auvray P. and Baudet M., $J$. Appl. Crystallogr 20 (1987) 111.

[7] Nagai H., J. Appl. Phys. 45 (1974) 3789.

[8] Neumann D. A., Zabel H. and Morkoc H., Appl. Phys. Lett. 43 (1983) 59.

[9] Neave J. H., Dobson P. J., Joyce B. A. and Zhang J., Appl. Phys. Lett. 47 (1985) 100.

[10] Auvray P., Baudet M. and RegrenY A., J. Cryst. Growth, 95 (1989) 288. 